\title{
Adaptive Impedance Control with Compliant Body Balance for Hydraulically Driven Hexapod Robot*
}

\author{
Addie IRAWAN**, Kenzo NONAMI***, Hiroshi OHROKU**, \\ Yasunaga AKUTSU** and Shota IMAMURA** \\ **Graduate School of Engineering, Division of Artificial Systems Science, Chiba University, \\ 1-33 Yayoi-cho, Inage-ku, Chiba-shi, Chiba, 263-8522, Japan. \\ E-mail: addie@graduate.chiba-u.jp

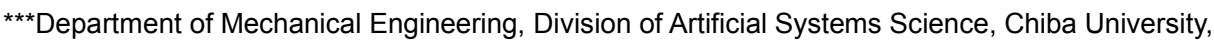 \\ 1-33 Yayoi-cho, Inage-ku, Chiba-shi, Chiba, 263-8522, Japan.
}

\begin{abstract}
This article described on the implementation of impedance control with the adaptive elements and compliant walking mechanism in hydraulically driven hexapod robot named COMET-IV. The main issue when applying impedance control in this robot is the body attitude stability during walking on the uneven terrain that contains of major soft surface. The impedance controller is derived for each leg from vertical motion changes. In addition self-tuning stiffness method is proposed as an adaptive element from the changes of the robot's body attitude vector (magnitude), to ensure the robot is self-adapted with the changes of stepped ground. On the other hand, compliant walking mechanism is designed for force-based walking trajectory and proposed impedance controller integration. The proposed controller and mechanism are verified by running the robot on the designed uneven terrain (extremely soft surface) in the laboratory using critical condition of side walking setting.
\end{abstract}

Key words: Impedance Control, Compliant Walking Mechanism, Magnitude Vector (Attitude), Self-Tuning Stiffness, Extremely Soft Terrain

\section{Introduction}

Recently most of the multi-legged robot designs are suited for uncertain terrain and environment even in different gravity like a space research project. The main issue is to make this multi-legged robot robust enough to overcome all the disturbances from the mentioned environment that could unstable the body posture. It is different to the manipulator system whereby legged/wheel/swim/flying robot walking considered much on overall body stabilization on designing the motion control for each driven element. On the other hand robot structures itself also influence its stability performances. In terms of legged-robots system structure and configurations, different scale and different number of degree of freedom (DOF) of each leg makes each research, and solutions has different goal and approaches. Such as ROBOCLIMBER [8], MECANT [9] and TITAN-XI [10] are different to the SILO [7] and AMRU [11] on a size, capacity and actuator system. Chiba University Operating Mine Detection Electronics Tools (COMET) version IV (COMET-IV) was developed and have a same actuator/driven system as [8], [9] and [10] but different on overall configuration of structures as shown in Figure 1.

${ }^{*}$ Received 22 Feb., 2011 (No. 11-0134) [DOI: 10.1299/jsdd.5.893]

Copyright $\odot 2011$ by JSME 

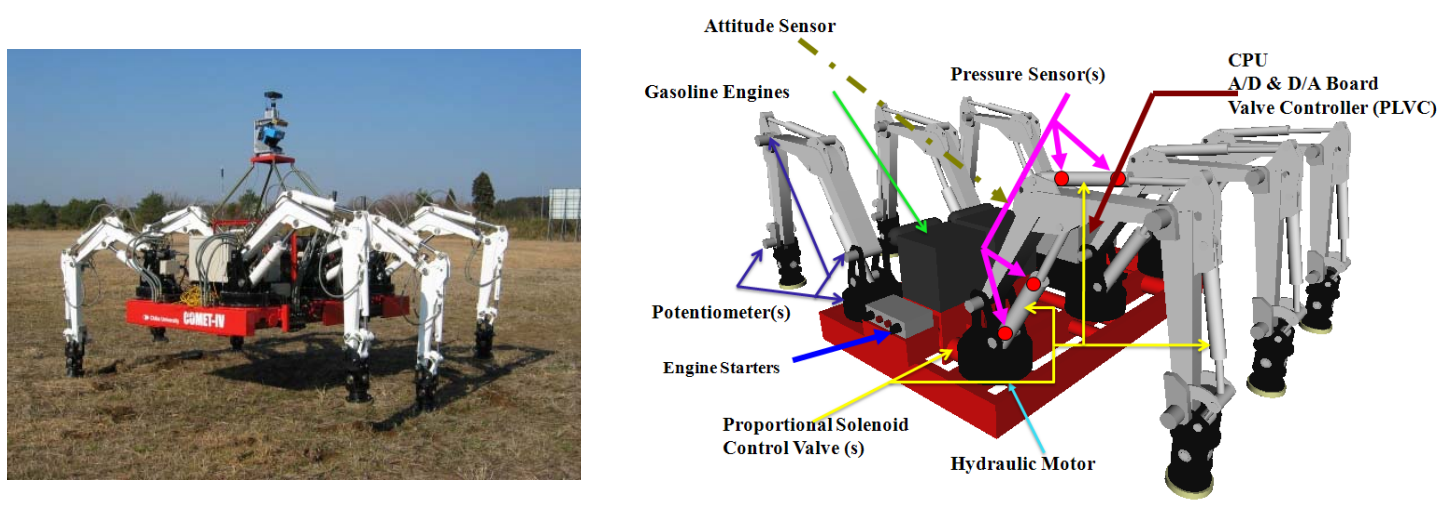

Fig. 1. COMET-IV features

As its name implies, the application is focused on the wide range of daunting task such as land-mine detection and as rescue tool at disaster area. For heavy weight machine such as MECANT, ROBOCLIMBER, TITAN-XI and COMET-IV, small scale (less than $5 \mathrm{~cm}$ height) an uneven terrain doesn't affect much on its walking. Therefore, walking on large scale an uneven terrain such as mountain area, slopes with high inclination, extremely soft-ground (wet soil, sand soil, etc.) and disaster area ( as that caused by an earthquake, etc.) becoming the main focus issue. In legged-robot motion control, several control methods have been proposed to solve the stability of overall body attitude, which involved position control tracking error, force control and attitude control with attached sensors feedback or vision system feedbacks. Moreover, sufficient force delivery on each foot is very important whereby overall balances will compensate for the body weight, weight of each leg and moment of inertia of the body during walking period. At this point, force control playing a main role other than attitude control.

Force control for legged-robot has been developed in various forms and techniques to comply with the leg configuration, walking pattern trajectory and body attitude of the robot. In hybrid force-position control such as proposed in [6], [8] and [9] each force output and position output need to be regulated at the same time to achieve better performance as required. This method needs a good force trajectory references if compare to the multi-stages or separated control approaches such as proposed in [7]. Force control can be designed with the direct method or indirect method according to the suitability in problem solving. Regarding to the walking on uneven terrain with a major soft surface issue, it is better to consider indirect force control method than direct force control method since the surface is dynamically changed. Indirect force control is suitable since it emphasized to guarantee a compliant behavior during the interaction between the end-effector and the contacted environment. Siciliano et. al. mentioned that the compliant control and impedance control is effective tools to achieve the indirect control of contact force. Compliant control is used to achieve a desired static behavior of the interaction and impedance control is used for dynamic behavior [5].

Large-scale dynamic change environment and extreme soft terrain are some of the factors that make a heavy weight robot, such as the COMET-IV, horizontally unstable. This kind of robot needs to maintain a horizontal stable range attitude angle $(\operatorname{roll}(\varphi)$ and $\operatorname{pitch}(\varnothing))$ at least in between $-1.5^{0} \ll[\varphi, \emptyset] \ll 1.5^{0}$ based on the body coordinate system (BCS) (Appendix A), where normal friction and vibration of the overall system are considered. This position guarantees that the gasoline oil is in the balance position to ensure proper fluid delivery and utilization in the pump system and engine. It is also important in mapping 
process when walking using a navigator system via the laser range finder (LRF). Moreover, it is important ensure each leg motion moved in the angle limitation as listed in Table A of Appendix A. A foot being swallowed by the ground could happen when the robot is walking on extremely soft terrain. Therefore, this article will describe the design and implementation of impedance control to overcome the mentioned problem. The derived impedance control than is adapted with proposed self-tuning stiffness method that varied corresponding to the vector of body attitude criterions; average of robot's body height and flow of motions of the robot's body posture. The proposed impedance controller is integrated with the proposed compliant walking mechanism and applied to the tripod force-based trajectory system. Verification is done in the laboratory with setup uneven terrain contains extremely soft surfaces. The experiment is done with the robot at the condition of setting as follows: tripod gait pattern, walking at a height $\mathrm{S}_{\mathrm{h}}=0.78 \mathrm{~m}$ above ground, a critical situation setting (shoulder at $0^{0}$ ), slow low motion mode (16s/cycle) and uneven terrain with maximum height difference of $\pm 12 \mathrm{~cm}$.

\section{Walking Trajectory and Pattern}

COMET-IV is designed with three types of walking pattern; tripod, pentapod and tetrapod with various gate patterns which is fixed gate pattern (right, left, front and back), transitional gate pattern via duty factor and omni-directional pattern, which is currently used with vision-based control. Concerning the previous research progresses, tripod walking pattern is a stable and faster walking pattern as shown in Figure 2.

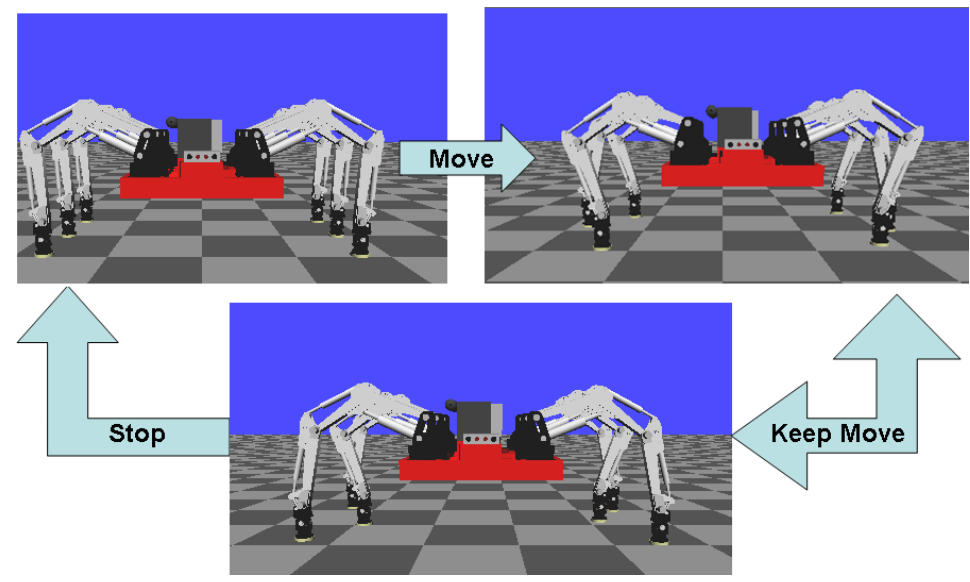

Fig. 2. Tripod pattern locomotion

In order to deal with uneven terrain, environment trailing trajectory (ETT) is proposed as a trajectory system with the tripod pattern as shown in Figure 3. This force-based trajectory walking is setup according to the following criteria:

1. Moved legs are contact to the ground until the center of gravity movement comes to end. The leg should trail environment pattern after a moved leg contacted with the ground.

2. Second is the maximum applied force should be reduced and controlled before swing phase is started to run the next step phase.

3. During grounding period, direction of force should follow the vertical direction of each leg's coordination where the total of force for each touching leg should be approximately or same as robot's body weight.

The foot trajectory pattern is designed with $90^{\circ}$ vertical push down to the ground as shown in Figure 4 in order to get precise vertical force delivery on foot. 


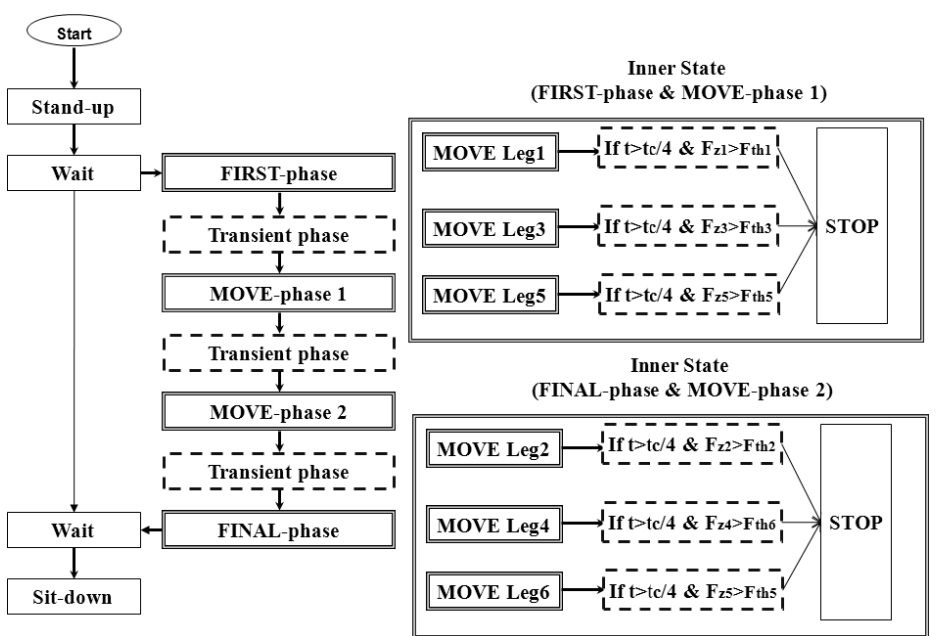

Fig. 3. General flow of ETT walking with tripod pattern

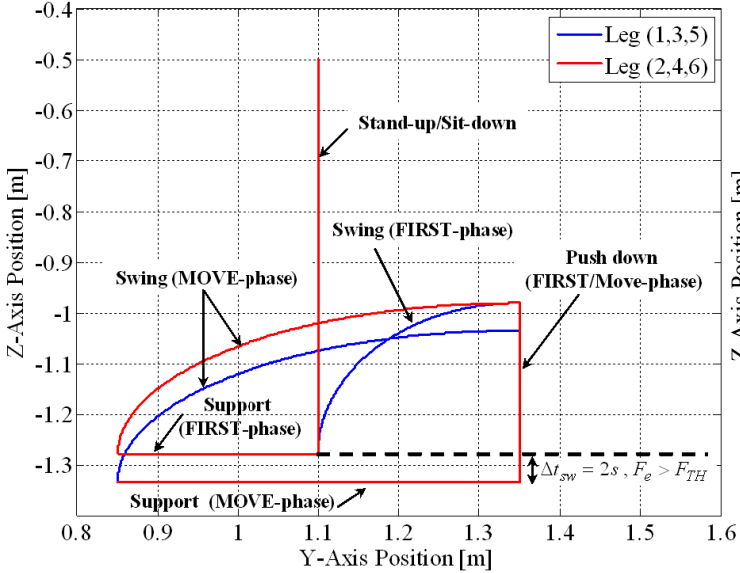

(a)

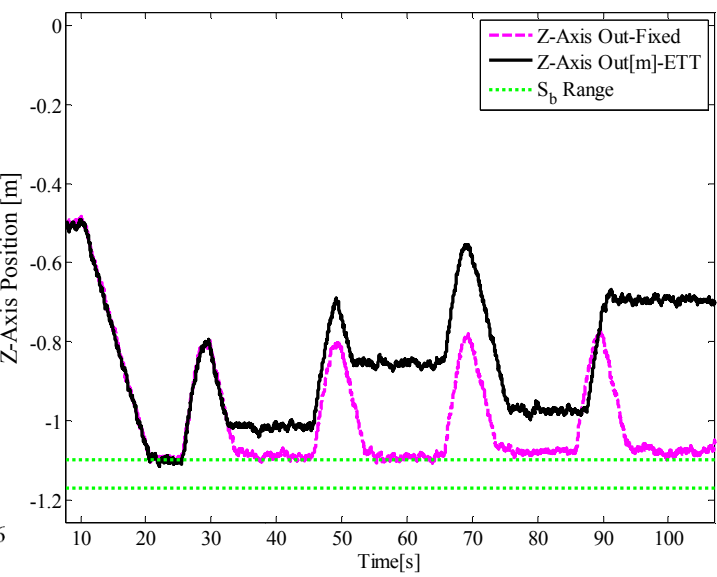

(b)

Fig. 4. ETT foot point movement pattern, (a) Reference signal (phase division) (b) Sample of experiment results $z_{o}(t)$ fixed/determined motion versus ETT motion

In ETT walking method, $1 k N$ of force threshold $\left(F_{T H}\right)$ is decided as an enough force for each leg to start patching and carry about 3 tons of robot's body during walking period.

\section{Compliant Walking Mechanism}

For robot stability on using proposed ETT system discussed in Section 2, compliant walking mechanism is proposed. This mechanism is design in order to solve the horizontal static stability on applying the ETT trajectory system. Practically, for static walking robot such as COMET-IV, the used of too large as well as low force on end-effector foot during the support phase period on some range of horizontal level is improper and technically unstable. As shown in Figure 5, inside the dotted line is considered as flat terrain for COMET-IV corresponding to the laboratory experimental of tripod walking with ETT only [2]. 


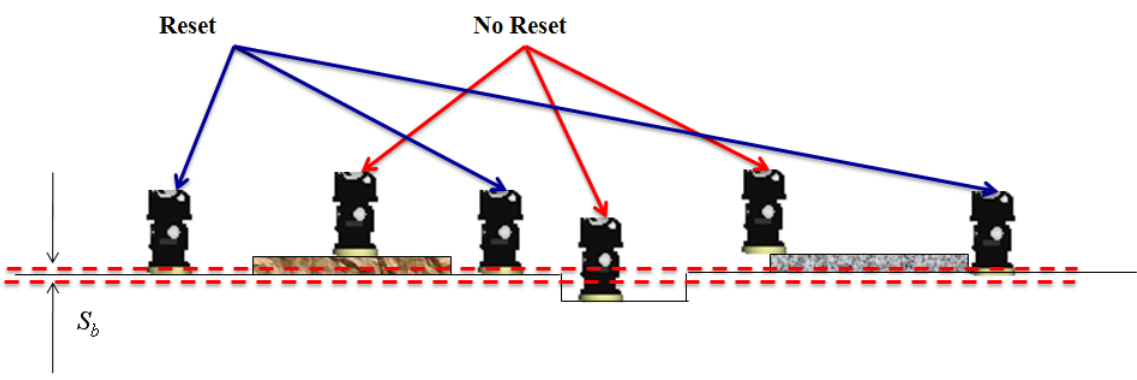

Fig. 5. Compliant mechanism concept for ETT control on uneven surface

Therefore, reset action needs to be done to avoid over pushing causing by redundant force effect. The dotted line range as shown in Fig. 5 can be determined by Equation (1) as follows:

$S_{b}=\left\{\begin{array}{c}Z_{0}+S H ; S_{b_{\text {min }}} \\ Z_{0}+S H+\ell_{z} ; S_{b_{\text {max }}}\end{array}\right.$

where $\ell_{z}$ is the maximum pushing range, $S_{b}$ which is set to $-0.7 \mathrm{~m}$ corresponding to the laboratory experimental of tripod walking with ETT only [2].

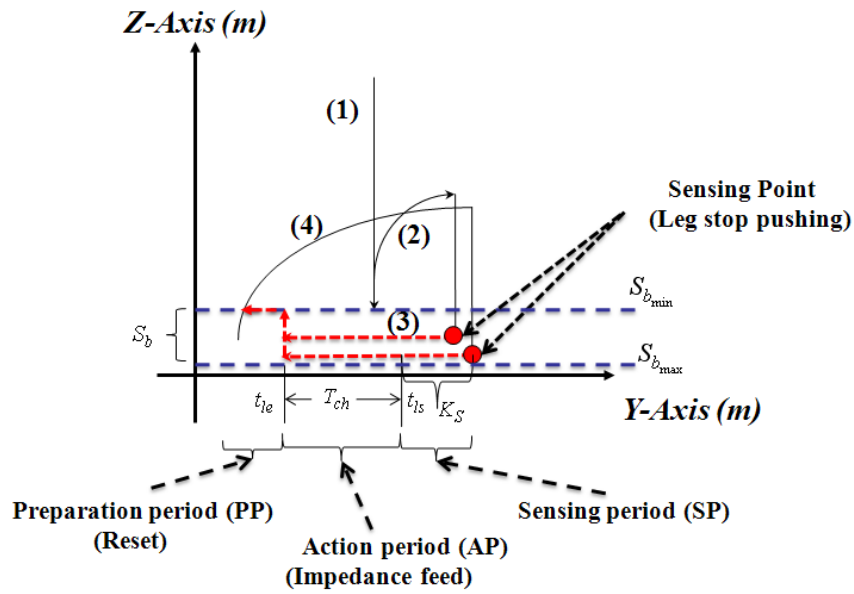

1-Stand-up, 2-Swing phase (First Step), 3-Support phase, 4- Swing phase (Next step)

Fig. 6. Proposed clusters of time for reset action

$T_{c h}$ and $K_{s}$ as shown in Figure 6 are tuning parameters for the cluster period setting proposed in [2]. Setting of proposed cluster period will affect the sensitivity and effectiveness of applied impedance controller.

\section{Impedance Controller Design}

In order to perform dynamic motion control during AP as discussed in Section 3, z-axis motion on each leg (vertical reaction) is considered for derivation of impedance control. Elastic model for each of the robot's leg is illustrated as Figure 7. 


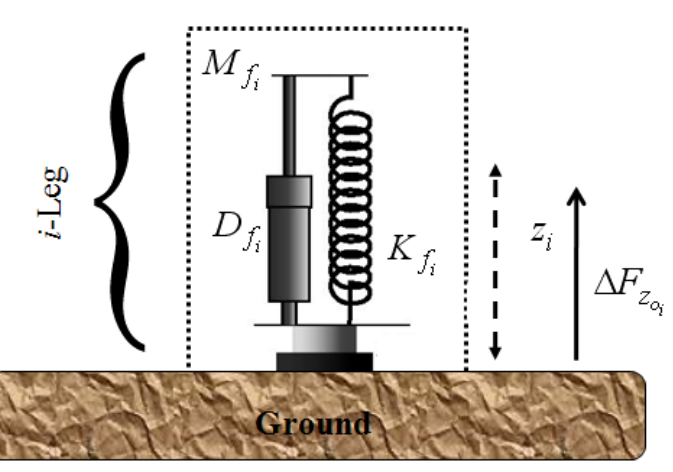

Fig. 7. Spring equivalent elastic model for robot's foot and environment

Vertical external force for each leg $\left(F_{\text {foot }}(z)=F_{z_{0_{i}}}\right)$ is derived via thigh and shank cylinders pressure, and torque of each joint of the leg (see Appendix B for $F_{\text {foot }}$ derivation) which is determined as follows:

$$
F_{z_{0_{i}}}=I_{1,3} F_{\text {foot }}
$$

where $i=1,2 . .6$ corresponding to number of leg and this $F_{z_{o_{i}}}$ is calculated whenever leg is touched on the ground (after $F_{z_{o_{i}}}>F_{T H}$ ) indicates by bit 1 since only end-effector force is considered for impedance controller input. Figure 8 shows the flow of end point forces (foot) derived from the pressure in cylinders at the thigh and shank of each robot leg and condition of feedback reading for applied impedance control.

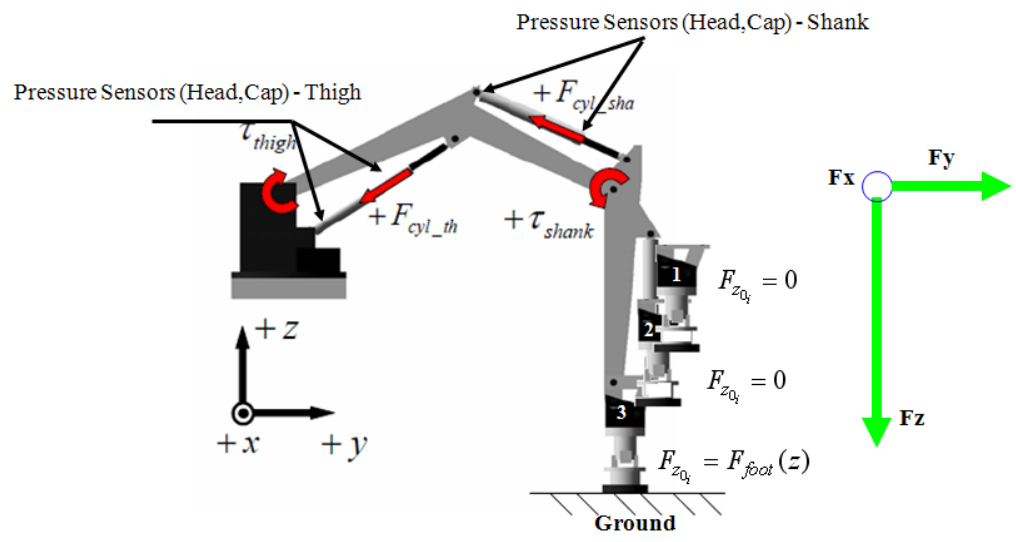

Fig. 8. Force coordination and configuration for each leg of robot and end-effector vertical force feedback condition

Thus impedance equilibrium for each robot's leg can be expressed as follows:

$-F_{z_{0_{i}}}=M_{f} \ddot{z_{i}}+D_{f} \dot{z_{i}}+K_{f} z_{i}$

where $z_{i}$ denotes as virtual vertical position for each leg. $M_{f}=1000 \mathrm{~kg}$, which is based on the actual weight for each leg and $K_{f}$ is a tuning parameter for each leg. On the other hand damping or viscosity coefficient $\left(D_{f}\right)$ of a controller is determined by applying Newton's Second Law where $F_{z_{o_{i}}}=0$, thus Equation (3) can be rearranged as follows: 
$\ddot{z_{i}}+\left(\frac{D_{f}}{M_{f}}\right) \dot{z_{i}}+\left(\frac{K_{f}}{M_{f}}\right) z_{i}=0$

Thus natural frequency and damping ratio of the impedance model can be written as follows:

$\omega_{0}=\sqrt{\frac{K_{f}}{M_{f}}}, \zeta=\frac{D_{f}}{2 \sqrt{M_{f} K_{f}}}$

In order to control the oscillation at the control output, critical damping (free vibration with damping) is chosen, $\zeta=1$. Thus desired $D_{f}$ for controller can be determined as follows:

$D_{f}=2 \sqrt{K_{f} M_{f}}$

Thus the new z-axis reference with impedance at the support phase period $\left(t_{c}\right)$ is expressed as follows:

$z_{I_{i}}\left(t_{c}\right)=Z_{R_{i}}\left(t_{c}\right)+z_{i}$

An Overview structure of derived impedance control for COMET-IV is shown as in Figure 9. Impedance control is an outer controller that dynamically changed the z-axis motion reference of the robot at $t_{c}$.

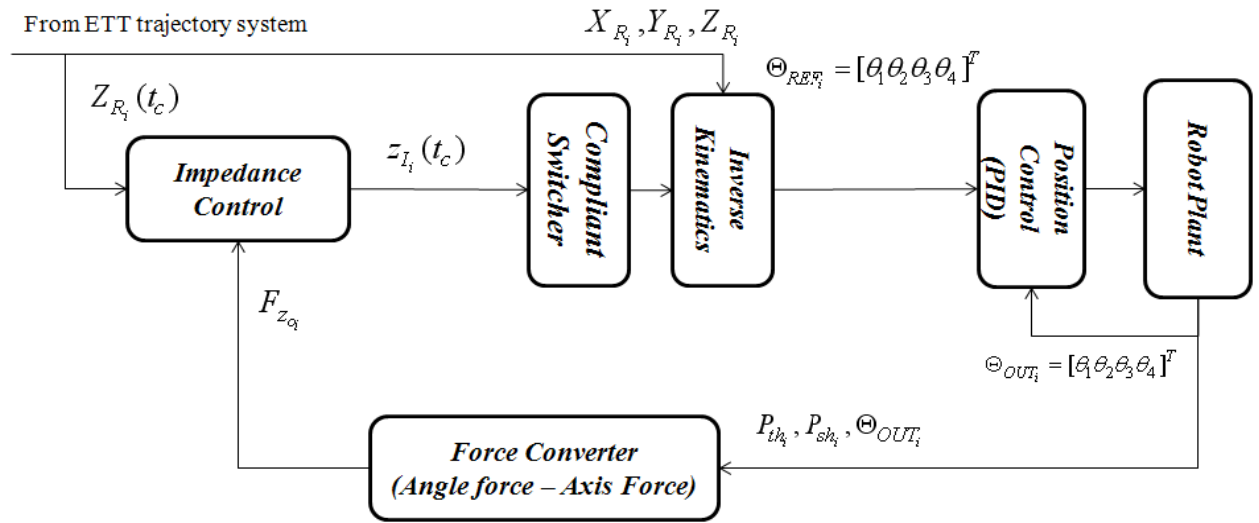

Fig. 9. General derrived impedance controller in the control module structure of the robot

\subsection{Self-tuning Stiffness}

As discussed earlier, $K_{f_{i}}$ is tuning parameter whereby $z_{I_{i}}\left(t_{c}\right) \propto K_{f_{i}}{ }^{-1}$ concerning the derivation previously and kinematics motions of the robot described in Appendix A. Therefore, for this hexapod robot, it is possible to consider the attitude feedback as a criterion to change the $K_{f_{i}}$. The attitude orientation and stability configurations for COMET-IV are shown in Figure 10. 


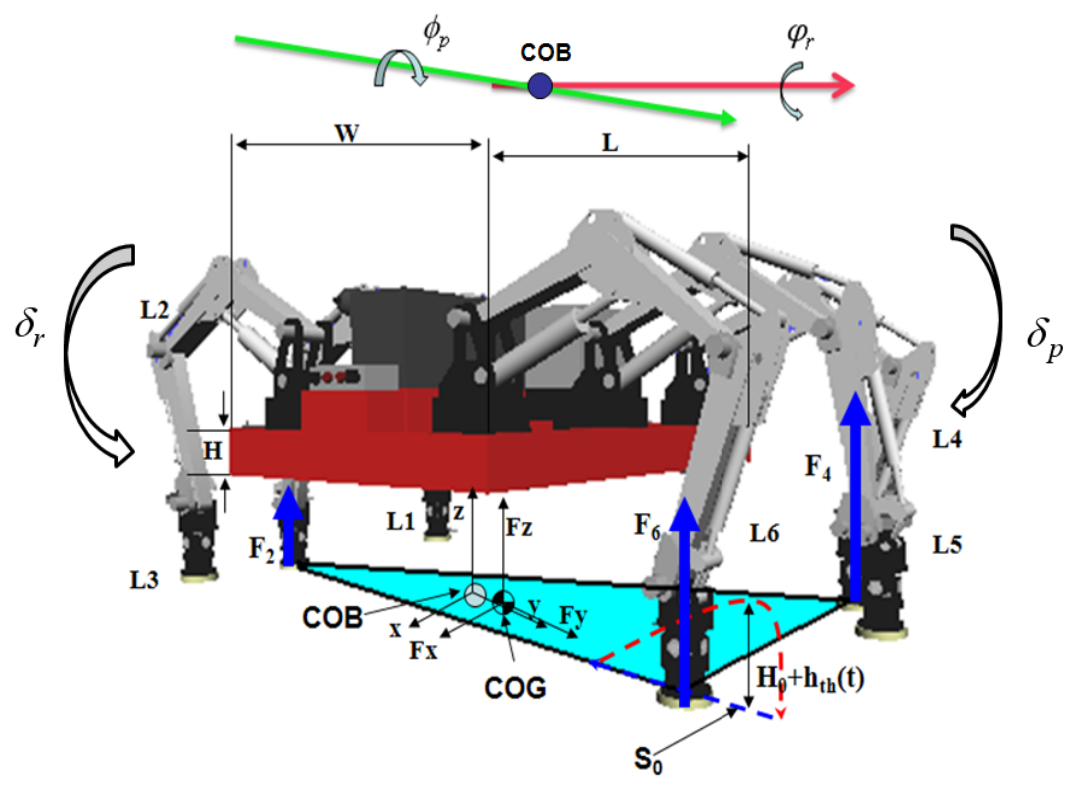

Fig. 10. Robot stability configuration and attitude orientation (euler angles)

As mentioned in Section 1, the horizontal stability for robot's body rotational is decided at a range $-1.5^{0} \ll[\varphi, \varnothing] \ll 1.5^{0}$ and average robot's body height error should be zero or nearly to zero. The average robot's body height error can be determined by differences of an ideal robot's body height $\left(B_{r}\right)$ and center of mass calculated as follows:

$\delta_{h}=\left\{\begin{array}{c}\sum_{r}-\frac{\sum_{i=a}^{c} z_{o_{i}} F_{z_{o i}}}{\sum_{i=a}^{c} F_{z_{o i}}} ; \text { Walking } \\ B_{r}-\frac{\sum_{i=1}^{6} z_{o_{i}} F_{z_{o i}}}{\sum_{i=1}^{6} F_{z_{o i}}} ; \text { Transient }\end{array}\right.$

where $B_{r}=\left|Z_{o}+S_{h}\right|, Z_{0}=-0.5 m$ is a fixed initial value for each leg vertical axis and $S_{h}$ is the ideal setup value for standing height of the robot which is both based on SCS (Appendix A). Therefore, the adaptive law for varying $K_{f_{i}}$ can be expressed as a relation as follows:

$K_{f_{i}} \propto\left[\delta_{h}, \delta_{r}, \delta_{p}\right]^{-1}$

where $\delta_{r}=0.5 L \varphi$ and $\delta_{p}=0.5 W \emptyset$ are the changes of robot's rotational posture angles when both angles at $-1.5^{0}>[\varphi, \emptyset]$ or $[\varphi, \varnothing]>1.5^{0}$ (Figure 10). In order to make $K_{f_{i}}$ changing in gradient proportional to the mentioned criterions, it is better to consider the exponentially changes of attitude feedback errors that containing magnitude vectors (arc of motions). Therefore, new expression of $K_{f_{i}}$ can be written as follows:

$K_{f_{i}}=K_{f_{o_{i}}}-p_{c} \chi=K_{f_{o_{i}}}-p_{c}\left(e^{-V_{a}}-1\right)$ 
where $K_{f_{o}}$ is initial stiffness value for each leg (pre-determined) and $V_{a}$ is a total error for the robot's body attitude feedbacks as follows:

$V_{a}=\delta_{h}+\sqrt{+\delta_{r}^{2}+\delta_{p}^{2}}$

$p_{c}$ is a positive tuning parameter for the range of stiffness changes and proposed cost function $\chi \rightarrow 0$ whenever $V_{a} \rightarrow 0$.

\subsection{Logical Attitude Control (Self-tuning Switching Function)}

Magnitude vector value in Equation (9) only applied for the disturbed leg(s) which is a leg that lower or higher (vertical position) than other legs during the support phase period. For undisturbed legs, $V_{a}=\delta_{h}$ is used since the range of $K_{f}$ for these legs are only proportioned to the $\delta_{h}$ rates. Therefore, a switching function is designed to control this situation with reference to the Euler angle of the robot in Figure 10 and robot's attitude counter as tabulated in Table 1. The truth table between rotational angles changes and leg positions are determined as tabulated in Table 2.

Table 1. Attitude counter indication based on legs status sequences (tripod)

\begin{tabular}{cccc}
\hline \hline$a_{c}$ & Status & $\begin{array}{c}\text { Leg } \\
\text { (Support) }\end{array}$ & $\begin{array}{c}\text { Leg } \\
\text { (Swing) }\end{array}$ \\
\hline \hline $\mathrm{K}$ & Walk & $2,4,6$ & $1,3,5$ \\
\hline $\mathrm{L}$ & Walk & $1,3,5$ & $2,4,6$ \\
\hline $\mathrm{M}$ & Transition & $1,2,3,4,6$ & - \\
\hline $\mathrm{N}$ & Transition & $1,2,3,4,6$ & - \\
\hline
\end{tabular}

Table 2. Relation of robot's body rotational angles and leg position (logical truth table)

\begin{tabular}{|c|c|c|c|c|c|c|}
\hline $\begin{array}{l}\text { Attitude } \\
\text { Angle }\end{array}$ & $\begin{array}{c}\text { Body } \\
\text { Angles }\end{array}$ & $\begin{array}{c}\text { Legs } \\
\text { position }\end{array}$ & Poles & $C_{a}$ & $C_{b}$ & $C_{c}$ \\
\hline \multirow{4}{*}{$\mathrm{K}$} & \multirow{2}{*}{$\varphi$} & $\mathrm{L} 1<\mathrm{L} 3, \mathrm{~L} 5$ & 1 & 1 & $\mathrm{NC}$ & $\mathrm{NC}$ \\
\hline & & L3<L1,L5 & 0 & $\mathrm{NC}$ & $\mathrm{NC}$ & 1 \\
\hline & \multirow{2}{*}{$\phi$} & $\mathrm{L} 5<\mathrm{L} 1, \mathrm{~L} 3$ & 1 & $\mathrm{NC}$ & $\mathrm{NC}$ & 1 \\
\hline & & $\mathrm{L} 5>\mathrm{L} 1, \mathrm{~L} 3$ & 0 & 1 & 1 & $\mathrm{NC}$ \\
\hline \multirow{4}{*}{$\mathrm{L}$} & \multirow[b]{2}{*}{$\varphi$} & $\mathrm{L} 4<\mathrm{L} 2, \mathrm{~L} 6$ & 1 & $\mathrm{NC}$ & 1 & $\mathrm{NC}$ \\
\hline & & L6 $<$ L2,L4 & 0 & 1 & $\mathrm{NC}$ & $\mathrm{NC}$ \\
\hline & \multirow{2}{*}{$\phi$} & L2>L4,L6 & 1 & $\mathrm{NC}$ & $\mathrm{NC}$ & 1 \\
\hline & & $\mathrm{L} 2<\mathrm{L} 4, \mathrm{~L} 6$ & 0 & $\mathrm{NC}$ & 1 & 1 \\
\hline
\end{tabular}

$C_{a}, C_{b}$ and $C_{c}$ as shown in Table 2 are denoted as logical attitude control outputs (supported legs respectively) for the desired switching of $V_{a}$ usage in Eq. (9). The Boolean expression for $C_{a}, C_{b}$ and $C_{c}$ with reference to the Table 2 are simplified via De Morgan's laws and written as follows:

$C_{a}=K \cdot(\bar{r}+r p)+L \cdot \bar{p}$

$C_{b}=K \cdot p+L \cdot(\bar{r}+r p)$ 
where NC (No changes) state is represented by bit $=0, p$ and $r$ are represented logical input for $\varphi$ and $\phi$ respectively with reference to the range $-1.5^{0} \ll[\varphi, \varnothing] \ll 1.5^{0}$. Thus self-tuning stiffness with logical attitude control for each leg of the robot is written as follows:

$K_{f}\left(t_{c}\right)= \begin{cases}K_{f_{o_{i}}}+p_{c}\left(e^{-V_{a}}-1\right) & C_{a}=1 / C_{b}=1 / C_{c}=1 \\ K_{f_{o_{i}}}+p_{c}\left(e^{-\delta_{h}}-1\right) & C_{a}=0 / C_{b}=0 / C_{c}=0\end{cases}$

where tuning reference and relation are due to the $z_{R_{n}} \propto{K_{f}}^{-1}$. The flow of proposed self-tuning impedance control is shown as Figure 11.

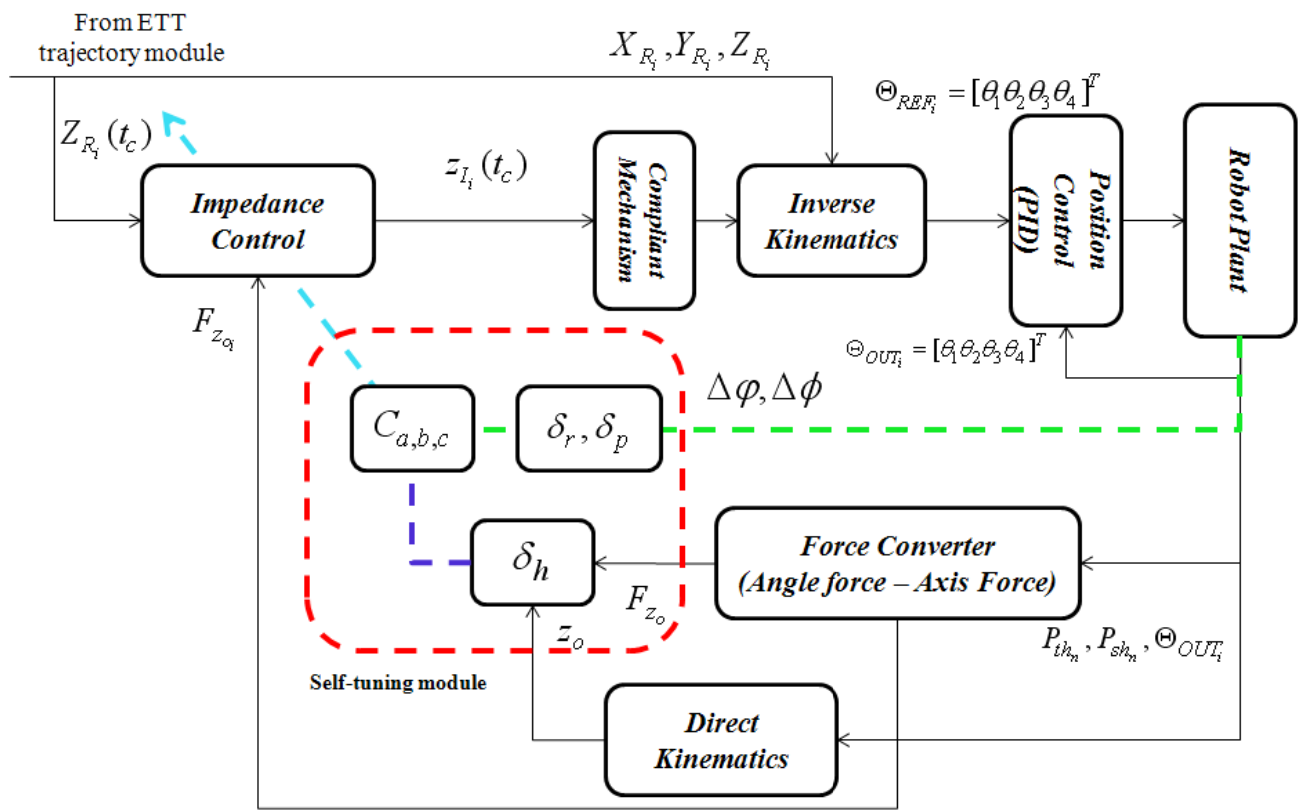

Fig. 11. Proposed implementation of self-tuning impedance control in robot control strucuture.

\section{Experiment and Results}

Fundamental tests are done for parameters tuning and others premier setting, which is the robot is running on an almost flat terrain surface (one layer of rubber plate) in the laboratory. In the test, $K_{f_{o}}$ and $p_{c}$ are tuning based on determined constrain of $z_{I_{i}}\left(t_{c}\right)$ at a range $-0.13 m>z_{I_{i}}\left(t_{q}\right)>0.08 m$ which is considering the motion signal limitation described in Appendix A. Moreover, the $K_{f_{o}}$ for each side leg (Leg 1,3,4 and 6) is decided to be 10 times lower than center leg (Leg 2 and 5) with reference to $F_{z_{o}}$ value between those legs. This decision to make sure each supported leg using an almost similar range of impedance during walking on almost flat terrain. $T_{c h}=K_{s}=3$ with reference to the setting in [2]. On the other hand, as shown in Figure 13, the same test also shows the integration between derived impedance control, and compliant walking mechanism did 
stabilized the ETT system. According to the robot's foot stepping signals (based on SCS) in Fig. 13, the integration shows horizontally stable for ETT system via performing some reset action in $S_{b}$ compare to the walking that only depends on ETT system.

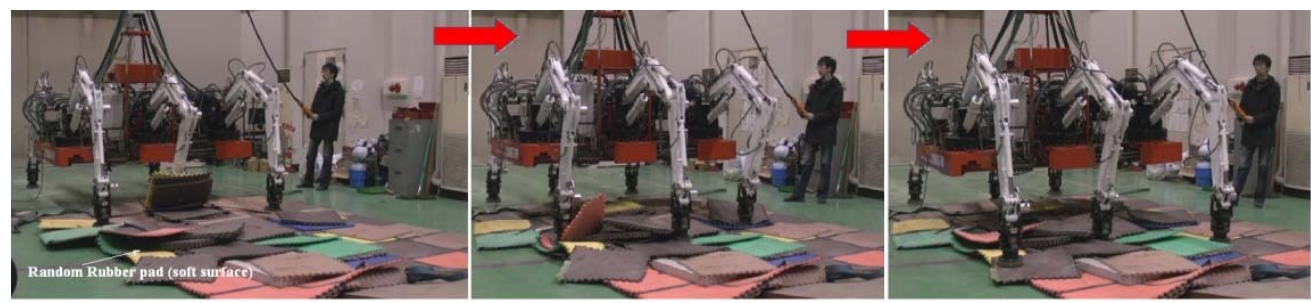

Fig. 12. Laboratory test setup (snapshots of COMET-IV running)

As mentioned in Section 1, verification for the proposed impedance control and adaptive impedance control are done in a laboratory as shown in Figure 12. About 3 layers of $4.2 \mathrm{~m}$ x $2.98 \mathrm{~m} \mathrm{~m} 0.02 \mathrm{~m}$ of rubber plate are used to representing soft terrain and randomly placed bricks with maximum $0.2 \mathrm{~m} /$ bricks including a multilayer rubber pad used to represent a soft surface cliff. For the experiments performed in this study, a proportional, integral, and derivative (PID) position controller is used (inner controller); $K_{p}, K_{i}$, and $K_{d}$ gain values of this controller for each joint are the same as those used for determined/fixed trajectory walking, and it is always being tuned properly to ensure the effectiveness of the impedance controller on each experiment running. The walking experiments are run in two times (right to left side walking); robot walking with Impedance controller (Imp) and robot walking with proposed adaptive impedance controller (self-tuning stiffness) (STImp).

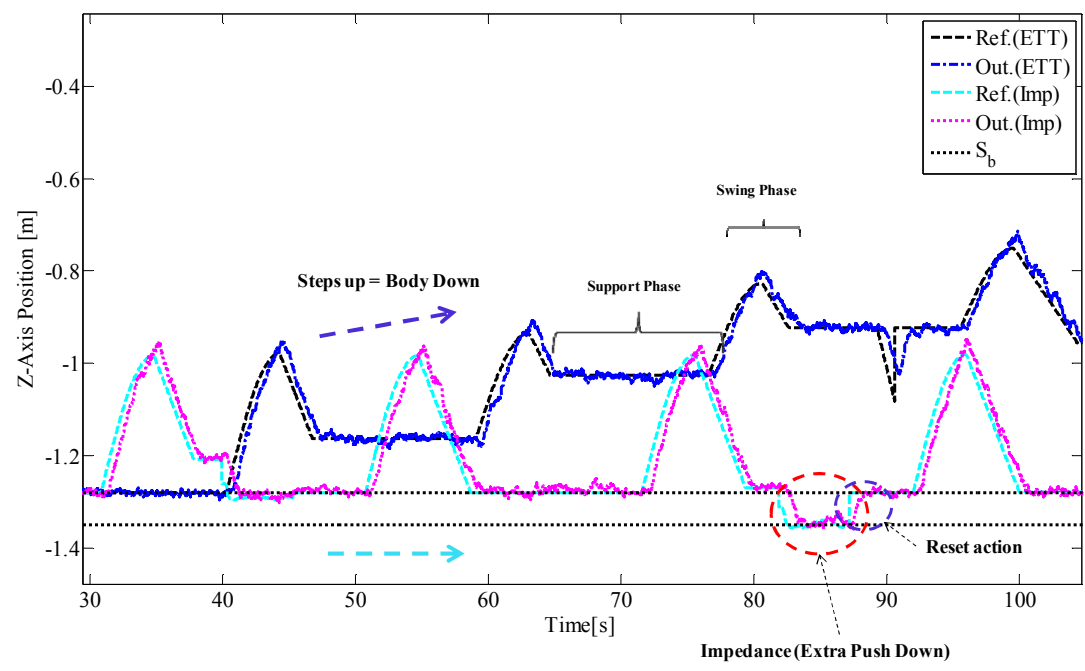

Fig. 13. Relation between walking with and without impedance control using ETT system (sample of Leg 2 z-axis motion) 


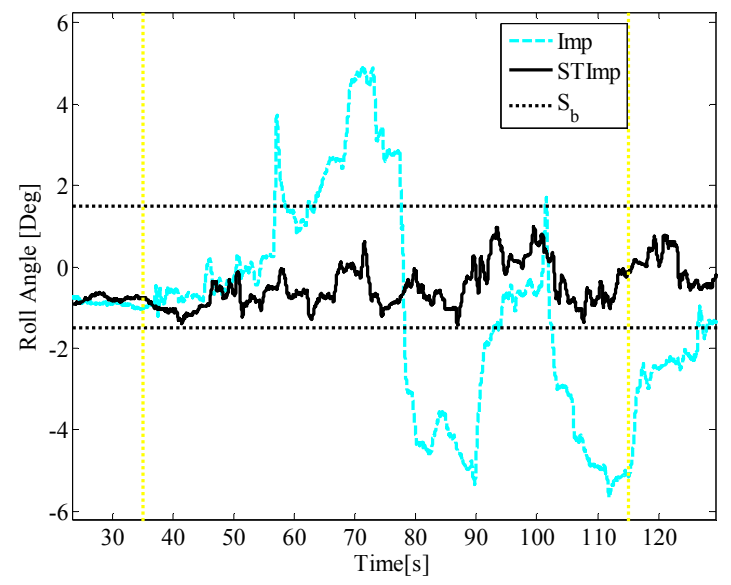

(a)

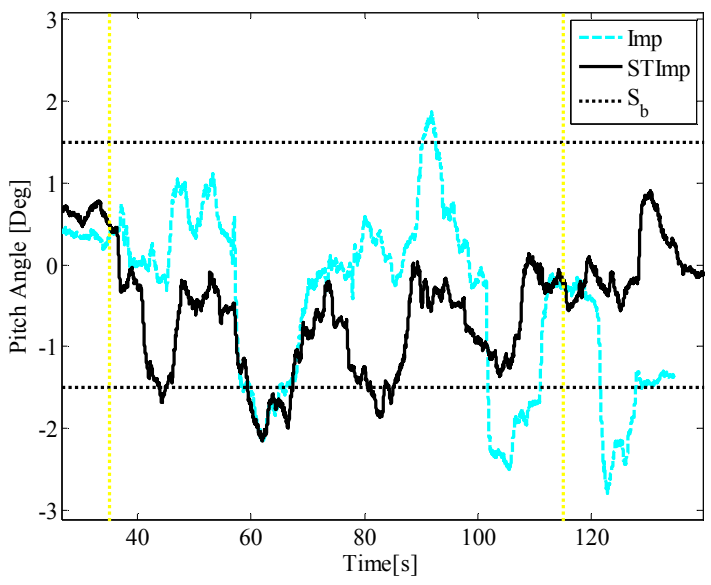

(b)

Fig. 14. Body rotational performances, (a) Roll angle output (b) Pitch angle output

Adaptation method improved the performance of robot walking on the soft surface as shown in Figure 14 and 15. Robot's body posture angles are in stable rage in STImp running to compare to the Imp running (Fig. 14). Furthermore, STImp running shows less body horizontal swinging as shown in Fig. 15 via body mass coordination (BMC) plot [3]. These performances are due to the STImp running that has self-tuning stiffness for each leg during each step of walking as shown in Figure 16.

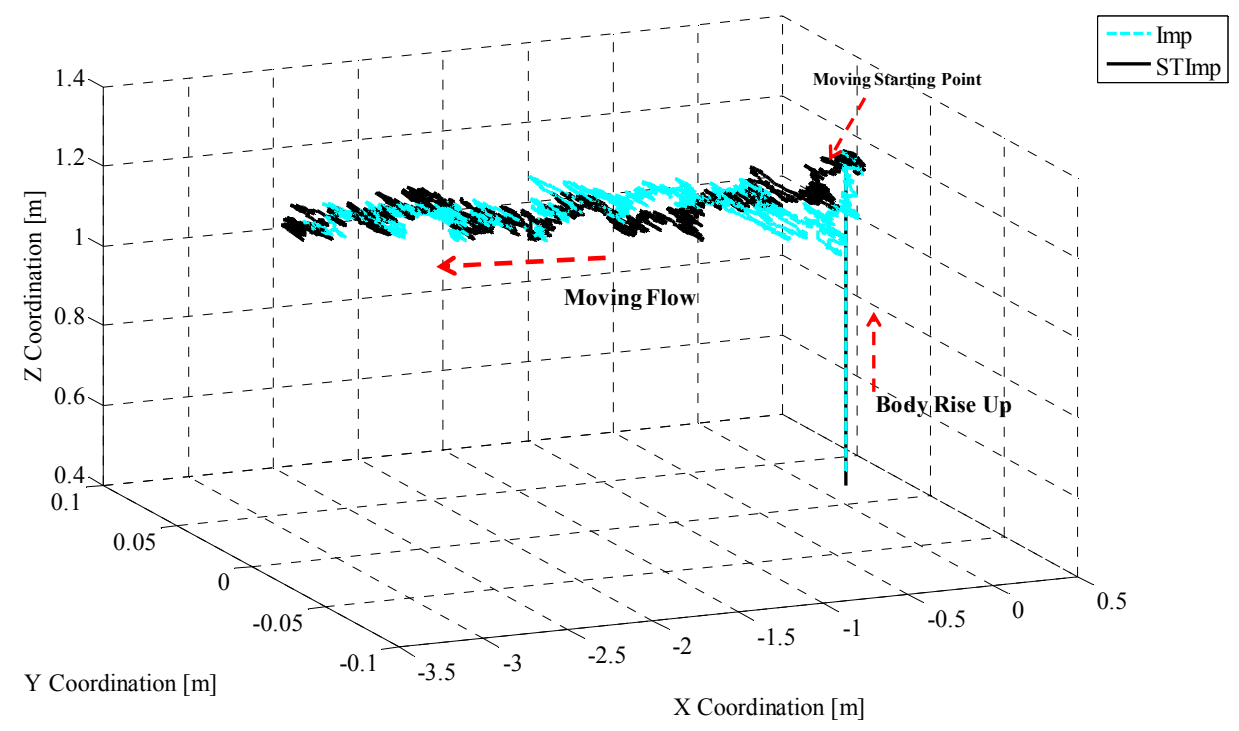

Fig. 15. BMC performances 

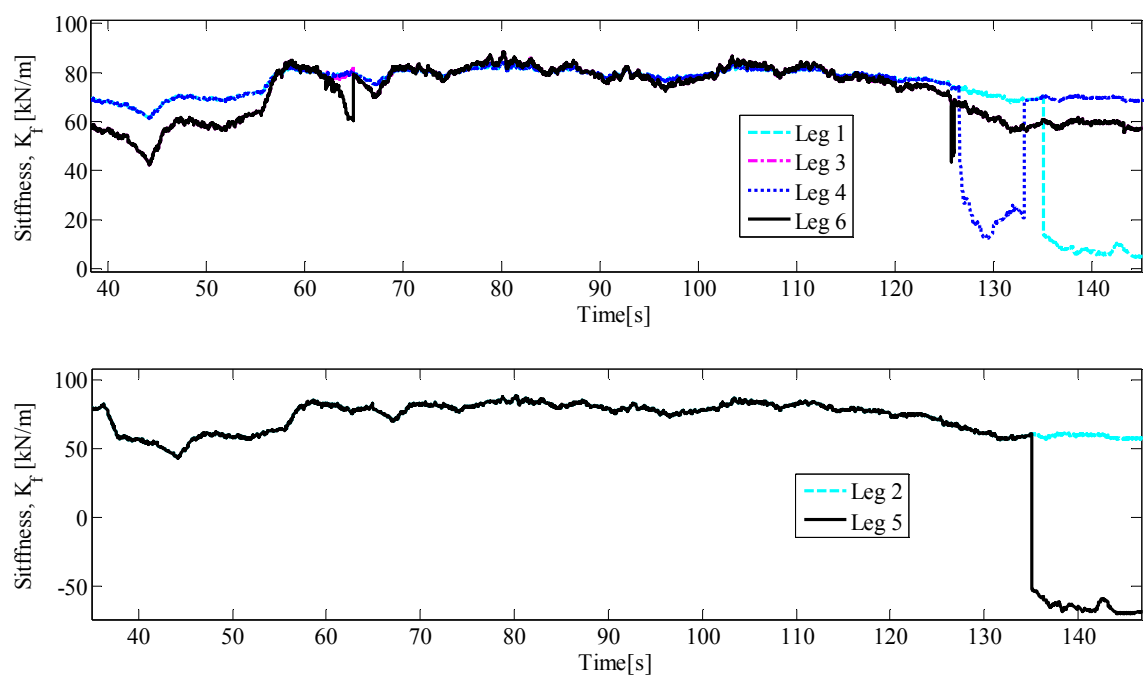

Fig. 16. Sample of leg's stiffness changes during walking period (Sample of STImp); top shows the stiffnesses of side legs and bottom shows the stiffnesses of center legs.
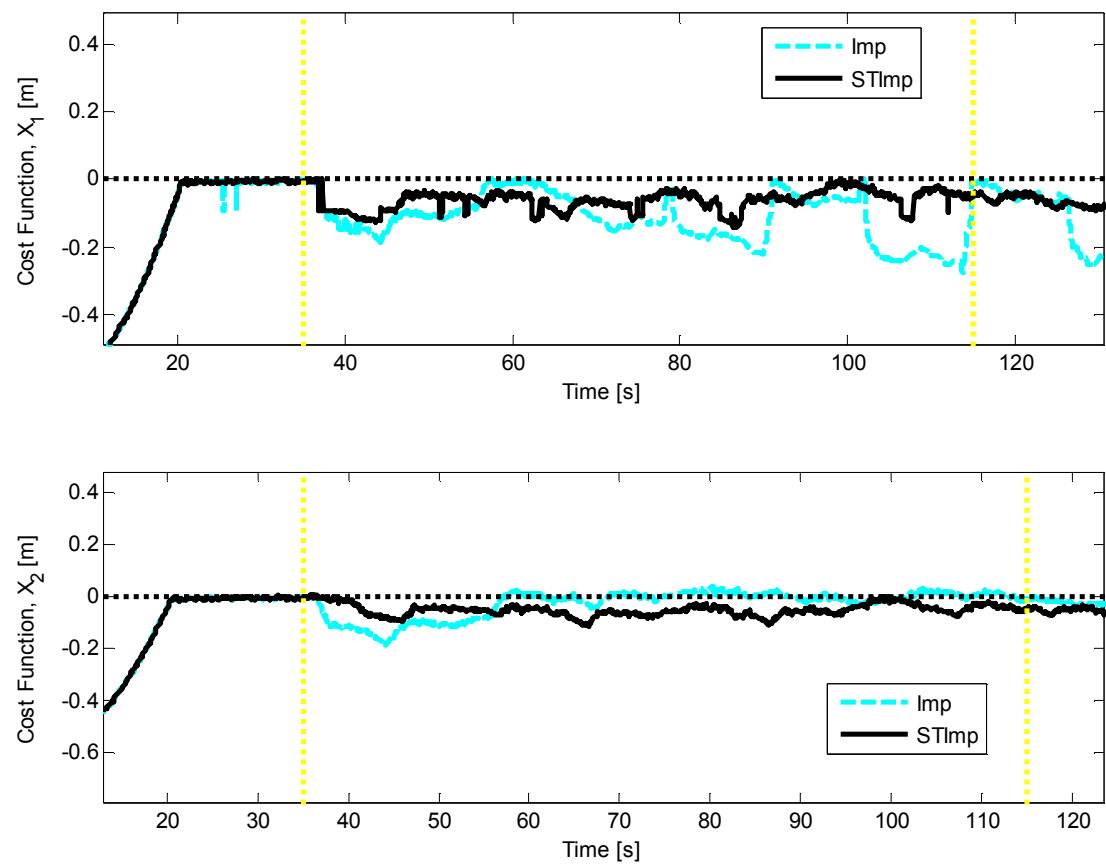

Fig. 17. Changes of $\chi$

The changes of $K_{f_{i}}$ as shown in Fig. 16 are proportional to the cost function evolution shows in Figure 17 where average performs $\chi_{1}=e^{-V_{a}}-1$ and $\chi_{2}=e^{-\delta_{h}}-1$ are nearly to zero for STImp running if compare to the Imp. Although $\chi_{2}$ for Imp running is near to zero compare to the STImp, the $\chi_{1}$ is worse by each steps in adverse to both results of $\chi_{1}$ and $\chi_{2}$ in STImp running which are more bettter in overall performances. Furthermore, side leg's motions (Leg 1,3,4 and 6) effect much on a body rotational angle changes thus playing a main role on balancing action (Fig. 16). For the setup environment situation, Imp running performed very critical walking with highly unstable on a roll angle (Figure 14(a)). 


\section{Conclusion}

In this paper, the adaptive impedance control of a robot leg's end-effector (supported legs) to solve the issue of walking on an extremely soft surface was presented. The basic difference between the classical impedance control and the proposed adaptive control is on the stiffness tuning, whereby the proposed impedance control applied a self-tuning stiffness that depends on the vector of robot's body attitude responses. Instead of estimating environment behavior and parameters, it is better to use the robot body criteria itself to automatically tuning the parameters of the impedance controller for hexapod robot such as COMET-IV. As discussed in Section 5, the proposed attitude vector gave impact to leg stiffness with supported by the proposed logical attitude control as switching function during robot walking on the setup environment. Moreover, it is also matched with proposed compliance switching and yet ETT system is stabled to be applied. However, the problem on small vibration during some foot placement whenever impedance is applied is still remained to be investigated. Further study also will be done to provide a better position controller as inner stage which one of the factor that can increase the effectiveness and efficiencies of impedance controller as an outer stage.

\section{Appendix}

\section{A. Leg Coordination and Kinematics}

Briefly, COMET-IV was designed with four degree of freedom (4-DOF) since it has four links for each leg [1]. The schematic diagram of body coordinate system (BCS) and shoulder coordinate system (SCS) is provided as Figure 18.

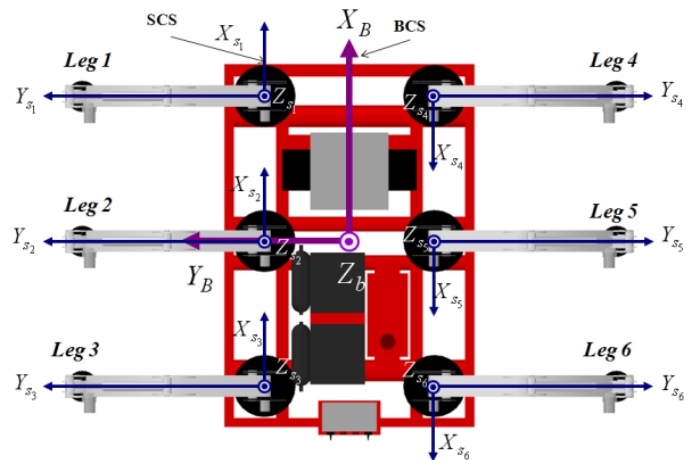

(a)

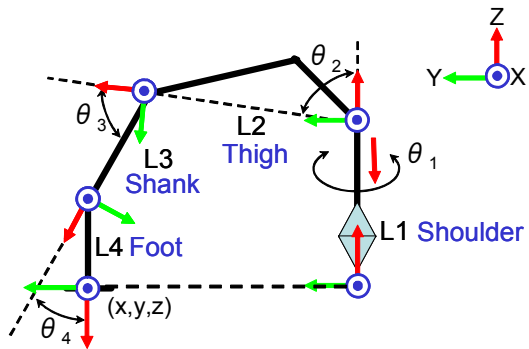

(b)

Fig. 18. Coordinate system used for COMET-IV, (a) SCS and BCS overall system (b) SCS for a leg

Table A: Link Angle Configuration (Limitations)

\begin{tabular}{cccc}
\hline \hline Joint & Link & Length $[\mathrm{m}]$ & Range $[\mathrm{deg}]$ \\
\hline \hline Shoulder & 1 & 0.00 & $\begin{array}{l}-120^{0} \ll \theta_{1} \ll 60^{0}(\text { Leg1-3) } \\
-60^{0} \ll \theta_{1} \ll 120^{0} \quad(\text { Leg4-6) }\end{array}$ \\
\hline Thigh & 2 & 1.13 & $53^{0} \ll \theta_{2} \ll 131^{0}$ \\
\hline Shank & 3 & 0.77 & $35^{0} \ll \theta_{3} \ll 144^{0}$ \\
\hline Foot & 4 & 0.39 & $-56^{0} \ll \theta_{4} \ll 108^{0}$ \\
\hline
\end{tabular}


For control purposes, a SCS as shown in Figure 18 (b) is derived and based on the kinematics of each leg, $\Theta_{\mathrm{REFi}}=\left[\begin{array}{llll}\theta_{1} & \theta_{2} & \theta_{3} & \theta_{4}\end{array}\right]^{T}$ to leg coordinate $\Gamma_{R E F i}=\left[\begin{array}{lll}x_{i} & y_{i} & z_{i}\end{array}\right]^{T}$ which is limited within specific range as shown in Table A. It should be noted that $i$ is represented the number of legs $(i=1 \ldots 6)$. The motion of each leg can be described using the following equations:

$x_{R_{n}}=\Delta \theta_{1}\left(L_{1}+L_{2} \Delta \theta_{2}+L_{3} \Delta \theta_{23}+L_{4} \Delta \theta_{234}\right)$

$y_{R_{n}}=\Delta \Phi_{1}\left(L_{1}+L_{2} \Delta \theta_{2}+L_{3} \Delta \theta_{23}+L_{4} \Delta \theta_{234}\right)$

$z_{R_{n}}=L_{2} \Delta \Phi_{2}+L_{3} \Delta \Phi_{23}+L_{4} \Delta \Phi_{234}$

Where $i, j$ and $k$ are a leg's joint tags which is $\theta_{i j k}=\sin \left(\theta_{i}+\theta_{j}+\theta_{k}\right)$ and $\Phi_{i j k}=$ $\cos \left(\theta_{i}+\theta_{j}+\theta_{k}\right)$. The inverse kinematics also derived to convert the leg coordinate (generated from trajectory sequences) to joint angles coordinates which is necessary to define the control input signal references. The inverse kinematics of each joint are described using the following equations:

$\theta_{1}=\tan ^{-1}\left(\frac{x}{y}\right)$

$\theta_{2}=\frac{\pi}{2} \tan ^{-1}\left(\frac{z-L_{4}}{\sqrt{x^{2}+y^{2}}}\right)-\tan ^{-1}\left(\frac{L_{3} \sin \theta_{3}}{L_{2}+L_{3} \cos \theta_{3}}\right)$

$\theta_{3}=\cos ^{-1}\left(\frac{x^{2}+y^{2}+\left(z-L_{4}\right)^{2}-L_{2}^{2}-L_{3}^{2}}{2 L_{2} L_{3}}\right)$

$\theta_{4}=\pi-\theta_{2}-\theta_{3}$

\section{B. Forces on Delivery to the Foot}

The force acting on the foot is estimated using the Jacobian matrix $(J)$ of the actual rotation of velocity (measured with the use of a potentiometer) for each joint of the robot's leg and the leg axis velocity. The Jacobian matrix of a leg is written as follows [6]:

$J=J_{3 x 4}=\frac{\partial \Gamma}{\partial \Theta}$

The force delivered to the foot through the torque of each link and pressure of the cylinder found on the thigh and shank can be expressed as follows:

$F_{\text {foot }}=J^{-T}\left(\tau_{c y l}+\tau_{g}\right)$

where $\tau_{g_{i}}=\sum_{s=a}^{c} J_{s} M_{s} g$ and $\tau_{c y l_{i}}=G_{t h_{n}} F_{c y l_{-} t h_{i}}+G_{s h_{n}} F_{c y l_{s} s h_{i}} . G_{t h_{i}}$ and $G_{t h_{i}}$ are the torque gains calculated based on Figure 19 [6]. 


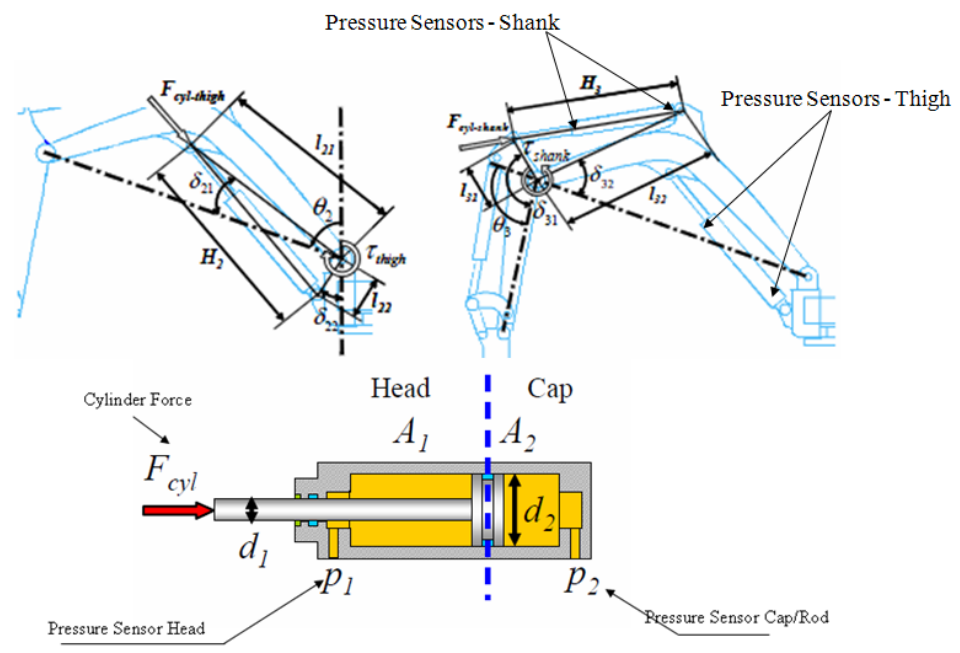

Fig. 19. Hydraulic cylinder configuration attached on each thigh and shank of robot Leg

\section{References}

(1) Oku, M., Yang, H., Paio, G., Harada, Y., Adachi, K., Barai, R., Sakai, S., Nonami, K. Development of Hydraulically Actuated Hexapod Robot COMET-IV -The 1st Report:System Design and Configuration. Proceedings of the 2007 JSME Conference on Robotics and Mechatronics, 2A2-G01, 2007.

(2) Irawan, A., Akutsu, Y., Nonami, K. Force-based Walking with Impedance Control for Hydraulic Driven Hexapod Robot. Proceeding of CLAWAR'2010: $13^{\text {th }}$ International Conf. on Climbing and Walking Robots and the Support Technologies for Mobile Machines, Nagoya, Japan, 66-72, 2010.

(3) Ohroku, H., Irawan, A., Nonami, K. A 3D modeling for Hydraulic-drive Hexapod Walking Robot using 3D Geometric Technique with distributed Numerical Model. ICGST-ARAS Journal, 9(1),31-39, 2009.

(4) Hogan, N. Stable execution of contact tasks using impedance control. Robotics and Automation. Proceedings. 1987 IEEE International Conference. Vol.(4),1047 - 1054, 1987.

(5) Siciliano, B., Villani, L. Robot Force Control.(Kluwer Academic Publishers, 1999).

(6) Oku, M., Koseki, H., Ohroku, H., Harada, Y., Futagami, K., Tran, D., C., Li, L., Lin, X., Sakai, S., Nonami, K. Rough Terrain Locomotion Control of Hydraulically Actuated Hexapod Robot COMET-IV (in Japanese). Proceeding of 2008 JSME Conf. on Robotics and Mechatronics (ROBOMEC 2008), 2008.

(7) Galvez, J.,A., Estremera, J., de Santos. P.,G. A new legged-robot configuration for research in force distribution. Mechatronics, 13, 907-932, 2003.

(8) Nabulasi, S., Armada, M., Montes., H. Multiple Terrain Adaptation Approach Using Ultrasonic Sensors for Legged Robots. Proceeding of Climbing and Walking Robots, 6, Part 6, 2006.

(9) Lehtinen, H. Force based motion control of a walking machine (VTT Publication, Espoo: VTT, 1994).

(10) Hodoshima, R. Doi, T. Fukuda, Y. Hirose, S. Okamoto, T. Mori, J. Development of a Quadruped Walking Robot TITAN XI for Steep Slope Operation - Step Over Gait to Avoid Concrete Frames on Steep Slopes. Journal of Robotics and Mechatronics, 19(1), 2007.

(11) Habumuremyi, J.,C., Baudoin, Y., Kool., P. Adaptative Neuro-fuzzy Control of AMRU-5, a six-legged walking robot. IARP WS Hudem'2004, Brussels, 16-18 Jun 2004.

(12) Erickson, D., Weber, M., Sharf., I. Contact Stiffness and Damping Estimation for Robotic Systems. International Journal of Robotics Research, 22(1),41-57, 2003.

(13) Terashima, K., Miyoshi, T., Mouri, K., Kitagawa, H., Minyong, P. Hybrid Impedance Control of Massage Considering Dynamic Interaction of Human and Robot Collaboration Systems. Journal of Robotics and Mechatronics, 21(1), 2009.

(14) Wang., X., Cui, Y.. Self-tuning Fuzzy Compensation based Adaptive Impedance Control for Robotic Machining. $2^{\text {nd }}$ International Conference on Computer Engineering and Technology (ICCET), 2010. 